\title{
BRANELESS BLACK HOLES
}

\author{
Edi Halyo ${ }^{a}$ †, Arvind Rajaraman ${ }^{b}$ and Leonard Susskind ${ }^{a}[$ \\ ${ }^{a}$ Department of Physics, Stanford University, Stanford CA 94305, USA \\ ${ }^{b}$ Stanford Linear Accelerator Center, \\ Stanford University, Stanford, California 94309 USA
}

\begin{abstract}
It is known that the naive version of D-brane theory is inadequate to explain the black hole entropy in the limit in which the Schwarzschild radius becomes larger than all compactification radii. We present evidence that a more consistent description can be given in terms of strings with rescaled tensions. We show that the rescaling can be interpreted as a redshift of the tension of a fundamental string in the gravitational field of the black hole. An interesting connection is found between the string level number and the Rindler energy. Using this connection, we reproduce the entropies of Schwarzschild black holes in arbitrary dimensions in terms of the entropy of a single string at the Hagedorn temperature.
\end{abstract}

\footnotetext{
${ }^{1}$ E-mail address: halyo@dormouse.stanford.edu

${ }^{2}$ E-mail address: arvindra@dormouse.stanford.edu

${ }^{3}$ E-mail address: susskind@dormouse.stanford.edu
} 


\section{Introduction}

Recently, a picture has been advocated [1] [2] of black hole entropy as arising from D-brane excitations. This naive D-brane picture of black hole entropy is known to be inconsistent when the black hole becomes massive enough for its Schwarzschild radius to exceed any microscopic scale such as the compactification radii. In [3] this was called the limit of a fat black hole. A typical example analyzed by Callan and Maldacena [2] and subsequently by Maldacena and Susskind[3] shows why this is so. In this 5-dimensional example, five branes and one branes are wrapped on a five torus and the system is given Kaluza-Klein momentum $N$ in one of the directions. The $\mathrm{D}$-brane picture says that the entropy is given in terms of a partition function

$$
Z=\prod_{n=1, \infty}\left(\frac{1+q^{n}}{1-q^{n}}\right)^{4 Q_{1} Q_{5}}=\sum d(N) q^{N}
$$

for a gas of $Q_{1} Q_{5}$ species of massless quanta. The integers $d(N)$ represent the degeneracy of the state with Kaluza-Klein momentum number $N$. For $N \rightarrow \infty$ keeping $Q_{1} Q_{5}$ fixed this gives an entropy

$$
S=\log d(N) \rightarrow 2 \pi \sqrt{N Q_{1} Q_{5}}
$$

This agrees with the classical black hole entropy. However, Maldacena and Susskind pointed out that the derivation is incorrect in the case of fat black holes because if $Q_{1}, Q_{5}$ and $N$ tend to infinity in fixed proportion, then one finds

$$
\log d(N) \rightarrow N \log N
$$

which does not agree witth the black hole entropy. Furthermore the same formula gives

$$
\log d(N)=\log \left(Q_{1} Q_{5}\right)
$$

for fixed $N$ and large $Q_{1} Q_{5}$. Thus the naive D-brane model fails to agree with $\mathrm{U}$-duality which requires symmetry among $Q_{1}, Q_{5}$ and $N$.

Maldacena and Susskind argued that a consistent theory could be formulated in which the $Q_{1} Q_{5}$ species are replaced by a single species and the level number $N$ is replaced by $N^{\prime}=N Q_{1} Q_{5}$. The entropy of the system is then carried by a single long string with a central charge six and a string tension $T \sim \frac{1}{g \alpha^{\prime} Q_{5}}$. Evidently, the picture which emerges from D-brane theory is that the black hole is a single string with a rescaled tension. A similar picture has been advocated in the past by one of us [4] and more recently by Tseytlin [5].

\section{$2 \quad$ A black hole in 5 dimensions}

We will analyze a 5-dimensional black hole made by wrapping $Q_{5} 5$-D-branes on $T^{5}$. This was analyzed by Maldacena in [6]. He showed that the near-extremal entropy of this configuration is given by the entropy of closed strings which live on the 5-brane and have zero winding and 
momentum. The central charge of these strings is given by $c=6 Q_{5}$ and their tension is $T=\frac{1}{g \alpha^{\prime}}$. However, this system suffers from the same problem described in the introduction. The correct entropy is only obtained in the limit $N=N_{L}=N_{R}>>c$ where $c$ is the central charge. However, for the near extremal case $N$ is small compared to $c$. In [6], it was conjectured that the correct configuration is one in which the string is fractionalized into strings with

$$
c^{\prime}=c / Q_{5}=6 \quad \alpha_{e f f}^{\prime}=g \alpha^{\prime} Q_{5} \quad N^{\prime}=Q_{5} N
$$

and the entropy formula is then correct. The rescaling of the string tension gives

$$
M=2 \sqrt{\frac{N}{g \alpha^{\prime} Q_{5}}}=2 \sqrt{\frac{N}{\alpha_{e f f}^{\prime}}}
$$

where $M$ is the deviation from extremality. The Hawking temperature becomes

$$
T=\frac{1}{\sqrt{g \alpha^{\prime} Q_{5}}}=\frac{1}{\sqrt{\alpha_{e f f}^{\prime}}}
$$

Note that the Hawking temperature appears to be the Hagedorn temperature associated with the effective string scale. We will show that the rescaling of the string tension can be understood as the blueshift of the energy of the string oscillations just as the Hawking temperature can be understood as the redshifted Hagedorn temperature [4].

The metric in the transverse 5-dimensions is given in [7]. We will use the notation of [8] with $\alpha=\sigma=0$. In this notation the equation for the metric is

$$
d s_{5}^{2}=-f^{-2 / 3}\left(1-\frac{r_{0}^{2}}{r^{2}}\right) d t^{2}+f^{1 / 3}\left[\left(1-\frac{r_{0}^{2}}{r^{2}}\right)^{-1} d r^{2}+r^{2} d \Omega_{3}^{2}\right]
$$

where

$$
f=\left(1+\frac{r_{0}^{2} \sinh ^{2} \gamma}{r^{2}}\right)
$$

The charge of the black hole is

$$
Q_{5}=\frac{r_{0}^{2}}{2 g} \sinh (2 \gamma) \simeq \frac{r_{0}^{2}}{g} \cosh ^{2}(\gamma)
$$

where the second relation is true in the near-extremal limit where $r_{0} \rightarrow 0, \gamma \rightarrow \infty$.

We are interested in the near horizon limit where the $(r, t)$ part of the metric will be seen to be two- dimensional Rindler spacetime. In this limit

$$
r \rightarrow r_{0}, f \rightarrow 1+\sinh ^{2} \gamma=\cosh ^{2} \gamma \equiv \lambda^{3}
$$

To bring the metric to the Rindler form, we rescale

$$
r^{\prime}=r \sqrt{\lambda} \quad r_{0}^{\prime}=r_{0} \sqrt{\lambda}
$$


Then

$$
\lambda r_{0}^{\prime}=r_{0} \cosh \gamma=\sqrt{g Q_{5}}
$$

Now expanding $r^{\prime}=r_{0}^{\prime}+y$, the near horizon metric takes the form

$$
d s_{5}^{2}=-\lambda^{-2} \frac{2 y}{r_{0}^{\prime}} d t^{2}+\frac{r_{0}^{\prime}}{2 y} d y^{2}+r^{2} d \Omega_{3}^{2}
$$

The proper distance $\rho$ from the horizon is then

$$
\rho=\int d \rho=\int \sqrt{\frac{r_{0}^{\prime}}{2 y}} d y=\sqrt{2 r_{0}^{\prime}} \sqrt{y}
$$

In terms of the proper distance, the coefficient of $d t^{2}$ becomes

$$
g_{00}=-\frac{\rho^{2}}{\lambda^{2} r_{0}^{\prime 2}}
$$

Rescaling

$$
\tau=\frac{t}{\lambda r_{0}^{\prime}}
$$

the $(\mathrm{r}, \mathrm{t})$ part of the metric becomes of the Rindler form

$$
d s_{5}^{2}=-\rho^{2} d \tau^{2}+d \rho^{2}
$$

The conjugate to $\tau$ is called the Rindler energy and will scale inversely to $\tau$. Hence we can write

$$
E_{R}=\lambda r_{0}^{\prime} \sqrt{\alpha^{\prime}} M=M \sqrt{g Q_{5} \alpha^{\prime}}
$$

Now we see a very interesting correspondence. Using eqn.(6) the Rindler energy can be simply written

$$
E_{R}=2 \sqrt{N}
$$

The Rindler energy is (apart from a factor of 2) just the square root of the oscillator number !

We can now use the well-known entropy for a string with oscillator number $\mathrm{N}$

$$
S=2 \pi \sqrt{\frac{c}{6}} 2 \sqrt{N}=2 \pi \sqrt{\frac{c}{6}} E_{R}=2 \pi \sqrt{\frac{c}{6}} M \sqrt{g Q_{5} \alpha^{\prime}}
$$

For $c=6$, we find the temperature

$$
T=\frac{1}{2 \pi \sqrt{g Q_{5} \alpha^{\prime}}}
$$

which is identical to the temperature found in [6] .

Note that the redshift factor connecting $\tau$ and $t$ is exactly the ratio of $\sqrt{\alpha_{\text {eff }}^{\prime}}$ to $\alpha^{\prime}$. Thus the picture is that of a fundamental string whose parameters have been rescaled due to redshifting. 


\section{Schwarzschild Black Holes}

We now show that the same reasoning holds for the Schwarzschild black hole in any dimension $D, 4 \leq D \leq 10$. The Schwarzschild solution in $D$ space-time dimensions is given by (we use the notations of [9])

$$
d s^{2}=-\frac{r-\hat{\mu}}{r} d t^{2}+\frac{r}{r-\hat{\mu}} d r^{2}+r^{2} d \Omega_{2}^{2}
$$

where

$$
\rho=r^{D-3} \quad \hat{\mu}=\frac{16 \pi G_{N} M}{(D-2) A_{D-2}}
$$

Here $A_{D-2}=\frac{2 \pi^{(D-1) / 2}}{\Gamma\left(\frac{D-1}{2}\right)}$ is the area of the unit sphere in $D-2$ dimensions. The black hole entropy is given by

$$
S=\frac{M^{(D-2) /(D-3)}}{4 G_{N}} A_{D-2}^{-1 /(D-3)}\left(\frac{16 \pi G_{N}}{D-2}\right)^{(D-2) /(D-3)}
$$

Close to the horizon, the proper distance to the horizon is given by

$$
R=\frac{2}{\sqrt{D-3}} \sqrt{y} \hat{\mu}^{\frac{1}{2(D-3)}}
$$

where $y$ is defined as $r=\hat{\mu}^{1 /(D-3)}+y$ near the horizon. The coefficient of the $d t^{2}$ term in the metric becomes

$$
\frac{r-\hat{\mu}}{r}=\frac{(D-3)^{2}}{4} R^{2}\left(\frac{16 \pi G_{N} M}{(D-2) A_{D-2}}\right)^{-2 /(D-3)}
$$

Rescaling $t$ to get the Rindler time $\tau$

$$
\tau=\frac{(D-3)}{2}\left(\frac{16 \pi G_{N} M}{(D-2) A_{D-2}}\right)^{-1 /(D-3)} t
$$

The relation between $E_{R}$ and $M$ is more subtle in this case 4 . The Rindler energy may be identified by requiring that it be conjugate to $\tau$, i.e. $\left[E_{R}, \tau\right]=1$. This can be written as

$$
1=\frac{(D-3)}{2}\left(\frac{16 \pi G_{N} M}{(D-2) A_{D-2}}\right)^{-1 /(D-3)}\left[E_{R}, t\right]
$$

We now use the fact that $t$ is conjugate to $M$ to write

$$
1=\frac{(D-3)}{2}\left(\frac{16 \pi G_{N} M}{(D-2) A_{D-2}}\right)^{-1 /(D-3)} \frac{\partial E_{R}}{\partial M}
$$

The Rindler energy is then

$$
E_{R}=M^{(D-2) /(D-3)} \frac{2}{D-2}\left(\frac{16 \pi G_{N} M}{(D-2) A_{D-2}}\right)^{1 /(D-3)}
$$


Now the relation derived in the previous section between $S$ and $E_{R}$

$$
S=2 \pi E_{R} \sqrt{\frac{c}{6}}
$$

gives the correct black hole entropy for $c=6$. Note that $c$ must be 6 in all dimensions. We have no deep understanding of why this is so, but at least in the cases of $D=4,5$, this is supported by the analysis of Tseytlin[5]. In addition, the identification of the square root of the string oscillator number $N$ with the Rindler energy $E_{R}$ works in all dimensions.

\section{$4 \quad$ String Length and Entropy}

We would like to make one more comment on the relation of entropy and string theory, which may be relevant to the question of black hole entropy. The point concerns a connection between the entropy carried by a string and its total integrated length.

Consider a free string thermally excited to average level $N$. Its entropy is $2 \pi \sqrt{\frac{N c}{6}}$.

Let us consider the total transverse length of the string. It is given in light-cone frame by

$$
L=\left\langle\int_{0}^{2 \pi} d \sigma \sqrt{\partial_{\sigma} X_{i} \partial_{\sigma} X_{i}}\right\rangle=2 \pi\left\langle\sqrt{\partial_{\sigma} X_{i} \partial_{\sigma} X_{i}}\right\rangle
$$

Now it is easy to prove that for a linear system like a free string that

$$
\left\langle\sqrt{\partial_{\sigma} X_{i} \partial_{\sigma} X_{i}}\right\rangle \propto\left\langle\partial_{\sigma} X_{i} \partial_{\sigma} X_{i}\right\rangle^{1 / 2}
$$

Furthermore, by the virial theorem,

$$
\left\langle\partial_{\sigma} X_{i} \partial_{\sigma} X_{i}\right\rangle=N \alpha^{\prime}
$$

where we have dropped quantum fluctuations.

Thus the mean length of string is

$$
L \sim N^{1 / 2} \sqrt{\alpha^{\prime}}
$$

On the other hand, the entropy of the string is $N^{1 / 2}$. Thus we find that the entropy per unit length of the string is a universal constant of order 1 bit per string length. If we think of the string as being subdived into segments of size $\sqrt{\alpha^{\prime}}$, then the entropy is the number of segments. This suggests that the horizon is occupied by string segments with a universal density of 1 segment per Planck area. 


\section{Discussion}

As far as we can tell, the entropy of fat black holes seems to have little to do with D-branes and more to do with string-like degrees of freedom. The entropy is dominated by configurations containing one long string. D-branes do seem to be important in certain limits, for example when the Kaluza-Klein momentum $\mathrm{N}$ is much larger than the other charges. This for example can be achieved when one of the compactification radii is much larger than the others and the black hole becomes a black string.

In the fat black hole limit, the same string like degrees of freedom seem to be relevant for near

extremal black holes as well as the opposite limit of Schwarzschild black holes. This suggests that they may also be relevant for other black holes.

\section{Acknowledgements}

The work of A.R is supported in part by the Department of Energy under contract number DE-AC03-76SF00515. L.S. was supported in part by NSF grant PHY 9219345. 


\section{References}

[1] A.Strominger and C.Vafa, "Microscopic Origin of the Bekenstein-Hawking Entropy", hepth/9601029

[2] C.Callan and J.Maldacena, "D-brane approach to Black Hole Quantum Mechanics", hepth/9602043

[3] J.Maldacena and L.Susskind," D-branes and Fat Black Holes", hep-th/9604042

[4] L.Susskind," Some Speculations about Black Hole Entropy in String Theory",hep-th/9309145

[5] A.A.Tseytlin," Extremal Black Hole Entropy from Conformal String Sigma Model", hepth/9605091

[6] J.Maldacena,"Statistical Entropy of Near-extremal Five-branes",hep-th/9605016

[7] G.Horowitz and A.Strominger,"Black Strings and p-branes", Nucl. Phys. B360,197,(1991)

[8] G. T. Horowitz, J. M. Maldacena and A. Strominger, "Nonextremal Black Hole Microstates and U-Duality", hep-th/9603109.

[9] A.Peet," Entropy and supersymmetry of D dimensional extremal electric black holes versus string states", hep-th/9506200 